Bolm Inst. oceanogr., S Paulo, 33(2):105-119, 1985

\title{
FORMA DA CORRELAÇÃO T-S DE MASSAS DE ĀGUA DAS REGIÕES COSTEIRA E OCEÂNICA ENTRE O CABO DE SÃO TOMË (RJ) E A ILHA DE SÃO SEBASTIÃO $(S P)$, BRASIL
}

\author{
Luiz Bruner de MIRANDA
}

Instituto Oceanográfico da Universidade de São Paulo (Caixa Postal, 9075, 01000 São Paulo, SP)

\section{Synopsis}

Characteristics of the structure and sea water properties on the continental shelf and waters adjacent to the continental slope, were studied based on the temperature-salinity (T-S) relationships. The water sampling was carried out with standard methods in a network of hydrographic stations worked in five-time intervals (5-10 days), from January 1970 through February 1971. The classification review of the water masses used under regional conditions confirm that the Subtropical-Deep (STDW), Subtropical (STW) and South Atlantic Central (SACW) Water Masses are different nomenclature for the same water mass; the latter is suggested to indicate a water mass with a thermohaline index given by $\left(20.0^{\circ} \mathrm{C} ; 36.36 \%\right.$, obsemed undemeath the Tropical Water Mass. This salinity value is slightly higher than the modified thermohaline index of the SACW $(36.20 \%$ \% ), and can be used with great simplification in volumetric studies in the area. Polynomial expressions of the mean T-S curve, whose coefficients were computed with the least squares method, were given for practical applications. From these applications, altemative methods for the determination of the thermohaline derivative and constant density ratio $\left(R_{0}=\alpha d T / B d S\right)$ were obtained.

Descriptors: Water masses, Continental shelf, Continental slope, Coastal zone, Oceanic zone, T-S Diagrams, R/V "Prof. W. Besnard", Southeastern coast: Brazil.

Descritores: Massas de ägua, Plataforma continental, Talude continental, Zona costeira, Zona oceânica, Diagramas T-S, N/Oc. "Prof. W. Besnard", Costa sudeste: Brasil.

\section{Introdução}

E um fato bem conhecido que a técnica de anālise de massas de água com o diagrama $\mathrm{T}-\mathrm{S}$, introduzida por Björn Helland-Hansen em 1916, fundamenta-se na estratificação vertical das massas de água envolvidas nos processos de circulação e mistura. Em trabalhos sobre as características oceanográficas ao longo da costa brasileira, esse método foi utilizado por vários pesquisadores (Silva, 1959; Emîlsson, 1961; Okuda, 1962; Mascarenhas et al., 1971, dentre outros), sendo identificadas as principais massas de água envolvidas na circulação costeira e oceânica.

A partir desses trabalhos, as investigações da região em estudo se intensificaram, revelando algumas de suas complexidades. O fenômeno da ressurgência costeira, notável ao largo de Cabo Frio, tem sua geração relacionada a processos

Publ. n. 631 do Inst. oceanogr. da Usp. de interação ar-mar, à circulação e às características geomorfológicas da linha da costa e do fundo submarino. Esse fenômeno, ao ocasionar uma rápida renovação das águas costeiras (Silva \& Rodrigues, 1966; Almeida \& Azevedo, 1970; Ikeda et al., 1974; Tanaka, 1977; Rodrigues, 1977; Magliocca et al., 1979; Mesquita et al., 1979), por água de origem oceânica de camadas mais profundas, faz com que a correlação T-S das águas da plataforma continental seja muito semelhante aquela obtida para a região oceânica adjacente.

Neste trabalho é analisado um conjunto de dados amostrados em estações oceanográficas distribuídas densamente nas regiões costeiras eoceânica, entre o Cabo de São Tomé e a Ilha de São Sebastião (Fig. 1), com o objetivo de apresentar expressoes analíticas de curvas $\mathrm{T}-\mathrm{S}$ de massas de água e um exame comparativo dessas curvas, com aquelas obtidas quando da classificação de massas de água do 
Atlântico Sul. Como conseqüência, é apresentado um método alternativo, em adição ao proposto por Schmitt (1981), para a determinação da razão de densida- de constante, $R_{0}$, à partir da derivada termohalina (dS/dT) calculada ao longo da curva T-S representativa para as condições médias da região em estudo.

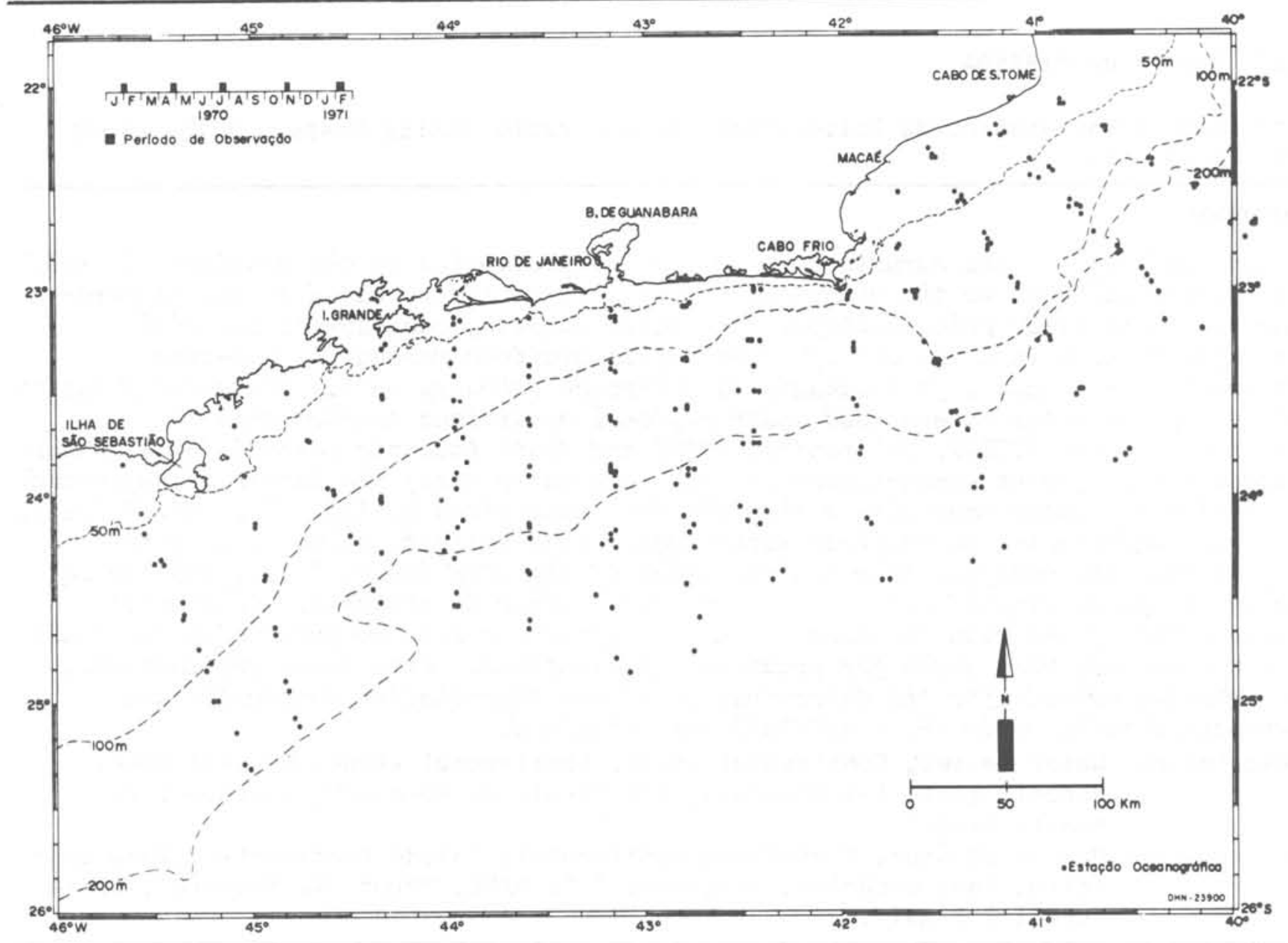

Fig. 1. Mapa da região: Cabo de São Tomé (RJ) a Il ha de São Sebastião (SP). Estão indicadas as posições das estações oceanogräficas realizadas nos cinco períodos de observação e as isőbatas de 50, 100 e $200 \mathrm{~m}$.

\section{Variāveis e metodologia}

Os cruzeiros oceanográficos, realizados a bordo do N/Oc. "Prof. W. Besnard", foram planejados de forma que a rede de estações oceanográficas ocupasse a mesma área e as estações, a mesma posição geográfica (Fig. 1). A região foi totalmente amostrada nos períodos de janeiro (fevereiro)/70, abril/70 e julho/70. A área de amostragem foi um pouco reduzida nos períodos de novembro/70 e fevereiro/ 71.

Em todas as estações oceanográficas, a coluna de água foi amostrada com garrafas-de-Nansen convencionais. A temperatura "in situ" (T) foi medida com termômetros de reversão protegidos. A salinidade (S) foi determinada por meio de medições da razão de condutividade elétrica $\left(R_{t}\right)$ com um salinômetro indutivo marca Beckman, modelo RS-7C, freqüentemente calibrado com água normal. $R_{t}$ foi convertida em salinidade, expressa em unidade de $\mathrm{g} / \mathrm{kg}(\% \circ)$, de acordo com as recomendações internacionais da época.

A profundidade ( $z$ ) de amostragem simultânea da temperatura e da salinidade foi obtida corrigindo-se, sempre que necessário, o comprimento do cabo lançado, com auxílio da profundidade termométrica e de acordo com o procedimento descrito por Miranda (1982).

Os valores do volume específico ( $\mathrm{V}$ P) foram calculados em função dos valores de $\mathrm{S}$ e $\mathrm{T}$ amostrados em profundidades, pela clássica equação de estado de Knudsen-Ekman e pela equação mais precisa de Chen \& Millero (1977).

$\mathrm{Na}$ equação de Chen \& Millero (op. cit.), o volume específico é determinado 
a partir das variäveis S, T e p, onde p é a pressão em bárias. Com os valores médios de $\mathrm{VP}^{\mathrm{P}}$, previamente obtidos para a região em estudo e que são concordantes aos determinados por Azevedo (1974) para as āguas da região sul e sudeste do Brasil, determinou-se, para intervalos de profundidade de 100 m e os correspondentes valores $\mathrm{p}(\mathrm{z})=\mathrm{gz} / \overline{\mathrm{VP}}$, a seguinte correlação entre a pressão, em bārias, e a profundidade, $z$, em metros:

$p(z)=0,100418 z+0,301379 \times 10^{-6} z^{2}$

Essa equação foi utilizada para a determinação da pressão quando do cálculo de VP com a equação de Chen \& Millero (1977).

As expressões polinomiais para as curvas T-S foram obtidas por meio de ajustes pelo método dos mínimos quadrados. Essas expressões analíticas foram usadas para cálculos da derivada termohalina (dS/dT) e para derivação de um método alternativo para o cáluculo da razão de densidade constante $\left(R_{\rho}\right)$.

\section{Trabalhos anteriores}

Dos estudos pioneiros de Deacon (1933; 1937) sobre as condições hidrológicas do extremo sul do Oceano Atlântico, verifica-se uma clara distinção entre as massas de água Tropical (AT) da camada de superfície, a Subtropical da Superfície (AST) e a Subtropical Profunda (ASTP); esta última é subjacente à āgua Tropical ao norte da Convergência Sub-tropical, razão pela qual recebeu essa denominação. De acordo com a prática usual, a denominação dessas massas de ảgua lembra o nome da região geográfica de sua formação. Os intervalos de variação da temperatura e da salinidade para a AT e a ASTP pela classificação de Deacon (op. cit.), estão apresentados na Tabela 1 .

Tabela 1. Intervalos de variação de temperatura, da sal inidade e da profundidade das Massas de Água Tropical (AT) e Subtropical Profunda (ASTP), de acordo com Deacon (1933; 1937)

\begin{tabular}{|c|c|c|c|c|c|c|c|}
\hline & $T$ & $\left({ }^{\circ} \mathrm{C}\right)$ & S & $(\% / 00)$ & $z$ & & m) \\
\hline AT & & $>23,0$ & & $>36,0$ & & $<$ & 145 \\
\hline ASTP & 10,5 & $-23,0$ & 34,85 & $=36,00$ & 145 & - & 510 \\
\hline
\end{tabular}

$\mathrm{Na}$ década seguinte, Sverdrup et al. (1942), utilizando dados coletados pela expedição alemã realizada no Oceano Atlântico a bordo do navio oceanográfico "Meteor" (1925-1927), retomaram o problema da classificação das massas de água desse oceano, com base no diagrama $\mathrm{T}-\mathrm{S}$. Nessa pesquisa, os pares $(\mathrm{S}, \mathrm{T}) \mathrm{da}$ camada de superfície $(0-150 \mathrm{~m})$, observados entre a região equatorial e o limite norte da Convergência Subtropical, não foram considerados, por não apresentarem uma correlação bem definida sobre o plano T-S. Entre a camada de superfície e a chamada Āgua Intermediāria Antārtica (AIA), cuja estrutura também foi pesquisada por Deacon em 1933, esses pesquisadores observaram uma boa coincidência entre a correlação T-S dos valores medidos em profundidade e a correlação T-S dos valores observados na superfície através da Convergência Subtropical. Assim, ficou confirmada a descoberta de Deacon (1933) de que, nessa região de convergência, formava-se uma importante massa de água, denominada por ele Subtropical.

Embora confirmando a ārea geogräfica de formação da massa de água Subtropical, essa massa de àgua, cuja profundidade atinge algumas centenas de metros, foi denominada Água Central do Atlântico Sul (ACAS). De acordo com Sverdrup et al. (1942), a correlação T-S é quase linear entre os pontos $\left(6,0{ }^{\circ} \mathrm{C} ; 34,5 \%\right.$ ) e $\left(18,0{ }^{\circ} \mathrm{C} ; 36,0 \%\right.$ \% $1 \%$. Uma envoltória que define a curva T-S média da ACAS para o Atlântico Sul, também è apresentada nesse trabalho (fig. 168, p. 626), permitindo que se observe claramente o desvio entre a curva média e a correlação 1inear.

Nos trabalhos pioneiros sobre as condições oceanográficas regionais ao longo da costa brasileira (Emílsson, 1959, 1961; Silva, 1959; Okuda, 1962), nos quais é utilịzada a correlação T-S para a caracterização das massas de água, pode-se identificar, na coluna de água fora da plataforma continental, as massas de āgua ASTP ou ACAS e a AT sobrepondo-se à AIA. No trabalho de Emílsson (1961), que abrange a faixa de latitude da região em estudo, foi adotada, praticamente, a mesma terminologia de Deacon (1933), notando-se, entretanto, as seguintes diferenças: a ASTP foi denominada simplesmente Água Subtropical (AST), possivelmente pelo aspecto regional de 
sua pesquisa, e os intervalos de temperatura e de salinidade para as massas de água AST e AT foram definidos por:

$10-20{ }^{\circ} \mathrm{C}, 35,0-36,0 \%$; $\mathrm{T}>$ $20,0^{\circ} \mathrm{C}$ e $\mathrm{S}>36,0^{\circ} \%$, respectivamente. Um esquema resumindo sobre o plano $\mathrm{T}-\mathrm{S}$ os principais resultados das pesqui- sas descritas nos parāgrafos precedentes è apresentado na Figura 2. Relativamente à definição da ASTP e da ACAS, as pesquisas de Deacon (op. cit.) e de Sverdrup et al. (1942) indicam uma coincidência dessas massas de água apenas na base da ASTP, cuja temperatura é de $10,5^{\circ} \mathrm{C}$;

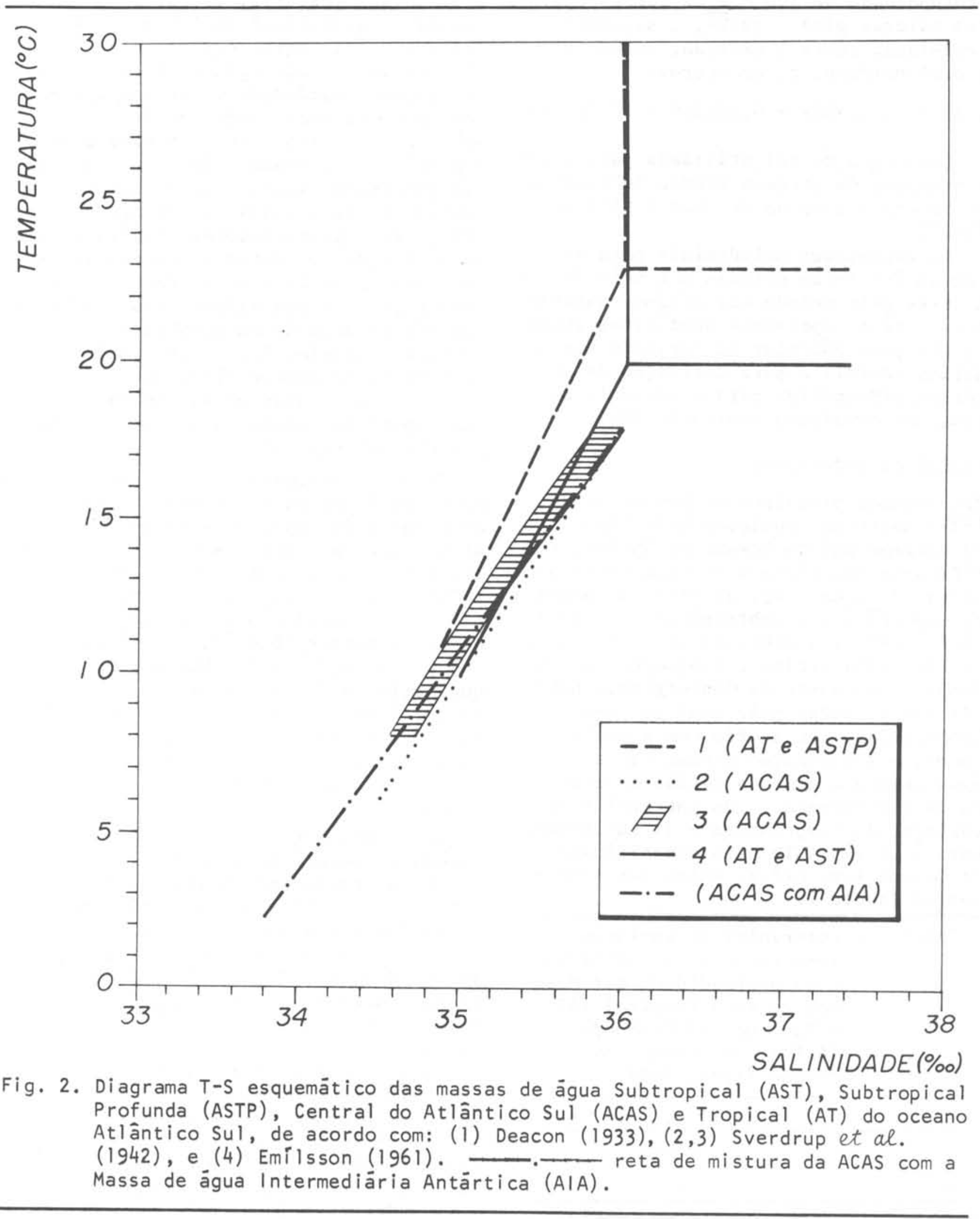


nesta temperatura, os desvios do valor médio de S $(34,85 \%$ \% são praticamente iguais a $\pm 0,08 \%$. Logo, de acordo com a classificação desses pesquisadores, a ASTP e a ACAS representam a mesma massa de água, apenas para temperaturas próximas a $10{ }^{\circ} \mathrm{C}$. Nessa Figura, verifica-se, também, que a AST definida por Emílsson (1961) e a ACAS de Sverdrup et al. (op. cit.) representam, praticamente, a mesma massa de água no intervalo de temperatura de 13 a $18^{\circ} \mathrm{C}$.

Do exposto, segue-se que a imagem sobre o plano T-S das características da coluna de água sobrejacente à AIA pode apresentar variações na correlação T-S, dependendo da região onde elas são examinadas. Entretanto, é evidente, do esquema da Figura 2, que, sob o ponto de vista regional, com modificações convenientes dos tipos de água representativos para os pontos extremos das massas de água ASTP, ACAS e AST, estas poderão ser consideradas como que diferentes designações de uma mesma massa de água.

\section{Caracteristicas regionais da correlação T-S}

Inicialmente são examinadas as características T-S da massa de água da camada de mistura das estações hidrográficas localizadas fora da plataforma continental; esta camada tem uma profundidade que varia entre 20 e $110 \mathrm{~m}$, sendo, os menores valores, encontrados em épocas de verão e nas estações localizadas no talude continental. De acordo com a terninologia de Deacon (1933), essa massa de água pode ser denominada de Ãgua Tropical (AT).

A comparação dos diagramas T-S espa1hados (Fig. 3), representativos da correlação dessas variáveis para os períodos de observação, indica que essa massa de água é sensîvel às variações sazonais dos processos meteorológicos. A maior estratificação que se observa no verão e no outono (Fig. 3A, B), contrasta com a maior homogeneidade dos períodos de inverno e primavera (Fig. $3 C, D$ ), onde a distribuição dos pontos sugere uma mistura quase isopicnal. Nessa Figura, observa-se, também, as seguintes características:

a - o máximo de salinidade (37,0 $37,1 \%$ é praticamente comum a todos os períodos, enquanto que o máximo de temperatura decresce de 28,5 a $24,0^{\circ} \mathrm{C}$. b - o intervalo de variação da salinidade (temperatura) é maior no verão, decrescendo de $1,25 \%$ \% $\left(8,0{ }^{\circ} \mathrm{C}\right)$ a $0,7 \%\left(2,5{ }^{\circ} \mathrm{C}\right)$, sendo o menor intervalo em novembro. Essa alteração na estratificação da camada de mistura, reflete-se igualmente na densidade, cujo intervalo de variação decresce de 2,0 a 0,3 em unidade de $\sigma_{\mathrm{T}}$.

c - os valores de S e T na base da camada de mistura variam entre 36,2 $36,5 \%$ e $20,0-22,0{ }^{\circ} \mathrm{C}$, respectivamente, e a densidade $\left(\sigma_{\mathrm{T}}\right)$ dessa camada é sempre inferior a $25,7 \mathrm{~g} / 1$.

Utilizando-se agora o conjunto de estações hidrográficas localizadas fora e, também, sobre a plataforma continental, examina-se a seguir as características T-S de toda a coluna de água. 0 diagrama T-S (Fig. 4) apresenta a correlação dessas variáveis para os períodos de janeiro (fevereiro)/70 e julho/70, representativos, em primeira aproximação, para as condições de verão e inverno, respectivamente; o espalhamento dos pontos nesse diagrama indica condições bastante diferentes entre esses dois períodos.

Para temperaturas acima de $15^{\circ} \mathrm{C}$, considerável número de pontos têm salinidades diferentes dos valores normais das massas de água de origens Tropical e Subtropical, sendo, esses pares (S, T), provenientes, principalmente, das estações oceanogräficas localizadas sobre a plataforma continental. Para valores de temperatura inferiores a $15{ }^{\circ} \mathrm{C}$ os pares $(\mathrm{S}, \mathrm{T})$ se distribuem com pequeno espa1hamento, e a configuração da curva T-S é pouco sensível à variação sazonal sendo, praticamente, coincidente com as caracteristicas originais da ACAS (Fig. 2). A distribuição sobre o plano T-S das observações dos demais períodos (abril/ 70, novembro/70 e fevereiro/71), mostra espalhamentos semelhantes aos da Figura 4. Uma diferença observada no mês de abril é a ocorrếncia de águas menos salinas, com mínimo próximo a $33,80 \%$, indicando uma maior influência de águas de origem continental.

0 espalhamento da distribuição T-S de cada um dos períodos indica que a plataforma continental estava inundada predominantemente por águas, transportadas pela Corrente do Brasil, de origens Tropical e Subtropical, em concor- 


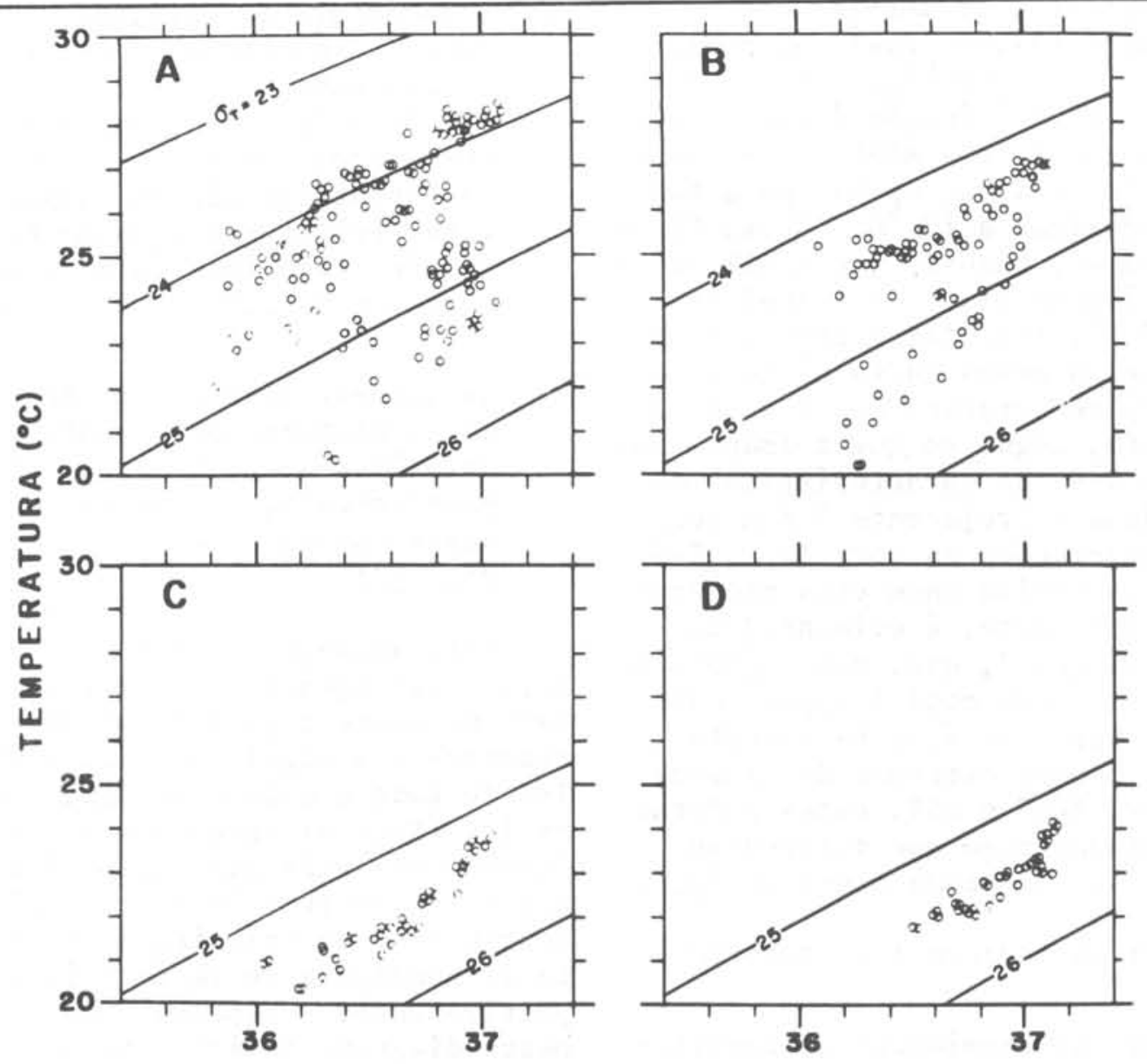

SALINIDADE (\%०)

Fig. 3. Diagrama $T-S$ da camada de mistura do conjunto de estações realizadas fora da plataforma continental ( $H>200 \mathrm{~m})$; janeiro(fevereiro) $/ 70$ e fevereiro/71 (A), abril (B), julho (C) e novembro (D). As profundidades de amostragem va-

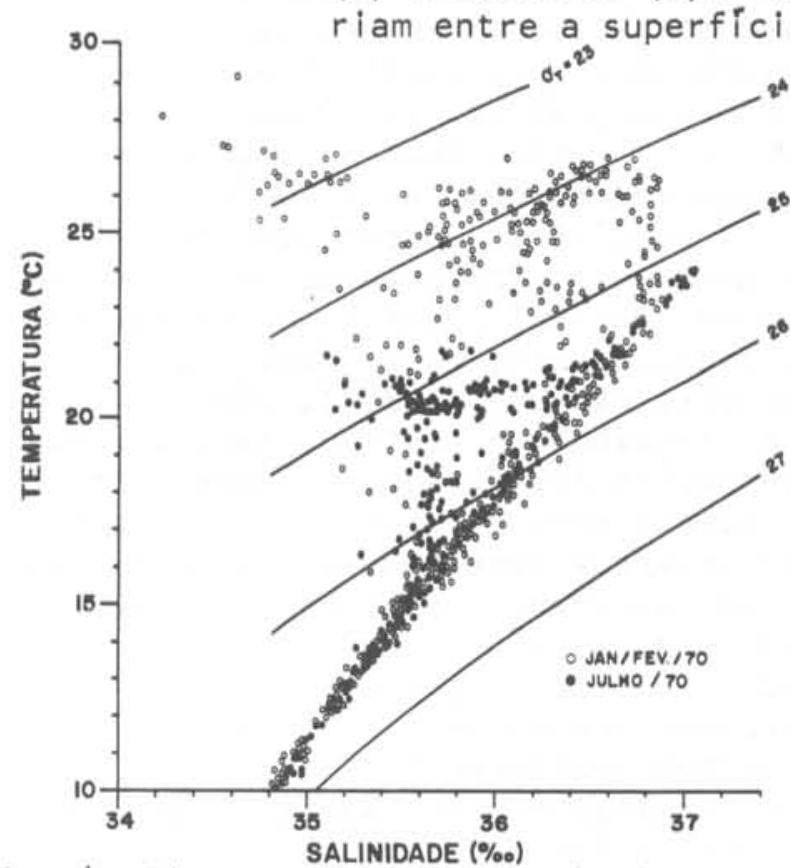

Fig. 4. Diagrama T-S espalhado das estações realizadas nos períodos de janeiro(feverei ro) $/ 70$ e jul ho/70, para temperaturas maiores que $10^{\circ} \mathrm{C}$. dância com os resultados pioneiros de Emilsson (1961) e Okuda (1962).

Forma analitica polinomial da curva $T-S$

Na construção do diagrama T-S espalhado para cada um dos períodos de observação, verifica-se que a correlação utilizada por Sverdrup et al. (1942) e Emílsson (1961) para classificar e definir a ACAS e a AST, também existe para pares (S, T) com S > $36,0 \%$ e $\mathrm{T}>20^{\circ} \mathrm{C}$. Este fato, já mencionado por Thomsen (1962) ao analisar as massas de āgua do Oceano Atlântico Sudoeste, sugere a determinação dos valores de $\mathrm{S}$ e $\mathrm{T}$ mais representativos para - Indice termohalino da ACAS para essa região do Atlântico Sul. Com efeito, aparentemente independente do trabalho de Thomsen (op. cit.), Mamayev (1975) apresenta o seguinte indice termohalino "modificado" para a ACAS: $\mathrm{T}=20,0{ }^{\circ} \mathrm{C}$ e $\mathrm{S}=$ $36,20 \%$. Esses valores são concor- 
dantes com os resultados das características termohalinas da base da camada de mistura obtida quando da análise do diagrama T-S espalhado (Fig. 3).

Para a seleção dos pares (S, T) observados na coluna de água, foram analisadas individualmente todas as estações oceanográficas abrangendo profundidades superiores a $200 \mathrm{~m}$, obtendo-se um total de 1040 pontos; neste total, estão incluídos todos os pares (S, T), cuja correlação quase linear se estende até a base da camada de mistura.

Os pontos assim obtidos foram analisados pelo método dos mínimos quadrados, de forma a ajustar-se, para a função $\mathrm{S}=\mathrm{S}(\mathrm{T})$, o melhor polinômio possível, cuja expressão genérica é dada na equação (2):

$$
S(T)=\sum_{i=0}^{k} B(i) T^{i} \quad(\% \circ)
$$

A variável $\mathrm{T}\left(\mathrm{em}{ }^{\circ} \mathrm{C}\right.$ ) foi tomada como independente, para que fosse obtida uma relação funcional mais conveniente para as aplicações präticas.

A representação da relação funcional entre as variáveis $S$ e $T$, na forma indicada na equação acima, foi sugerida por Bernardi Jr. (1969), num estudo preliminar sobre um método estatístieo para controle de dados oceanográficos.

Os coeficientes polinomiais $[B(i)]$ da equação (2) foram determinados para cada um dos períodos de observação e também para o conjunto total de pontos selecionados (1040). Estes coeficientes são apresentados na Tabela 2 e são vâlidos para a temperatura variando entre 6 e $24^{\circ} \mathrm{C}$; os polinômios correspondentes representam analiticamente as curvas $\mathrm{i}-\mathrm{S}$ médias dos períodos de observação.
A representação geométrica dessas curvas (Fig. 5A), por um número discreto de pontos, indica que o alinhamento destes é quase independente da época de observação, se considerarmos apenas os pontos com temperatura inferior a 20 $21{ }^{\circ} \mathrm{C}$. Esse fato sugere que a influência dos processos de interação ar-mar, alterando as características termohalinas na escala de tempo sazonal, é praticamente desprezível sob a base da camada de mistura (posição superior da termoclina permanente), identificada por esse valor de temperatura,

A curva T-S média, determinada à partir de todos os pares (S, T), cujos coeficientes polinomiais são dados na última linha da Tabela 2 , está representada geometricamente na Figura 5B. A envoltória dessa curva, representada por linhas tracejadas, indica o desvio médio quadrático $( \pm \varepsilon)$. Nessa Figura apresenta-se, também, a envoltória à curva média da ACAS determinada por Sverdrup et al. (1942) e, como se observa, as características termohalinas médias da região em estudo estão contidas dentro dos $1 i-$ mites originais definidos para a ACAS; esses resultados são apresentados quantitativamente na Tabela 3. Portanto, confirmando resultados anteriores (Okuda, 1962), a ACAS está presente na região em estudo, justificando-se adotar a classificação original de Sverdrup et al. (op. cit.), para a designação dessa massa de água.

De acordo com Mamayev (1975) o indice termohalino "modificado" da ACAS, representando as características dessa massa de água ao longo da costa leste da América do Sul, é dado por $\mathrm{T}=20^{\circ} \mathrm{C}$ e $\mathrm{S}=36,2 \%$. Conseqüentemente, à vista dos resultados obtidos anteriormente, têm-se, associado a esse valor de temperatura, um valor médio de salinidade

Tabela 2. Coeficientes da curva T-S sob a forma polinomial

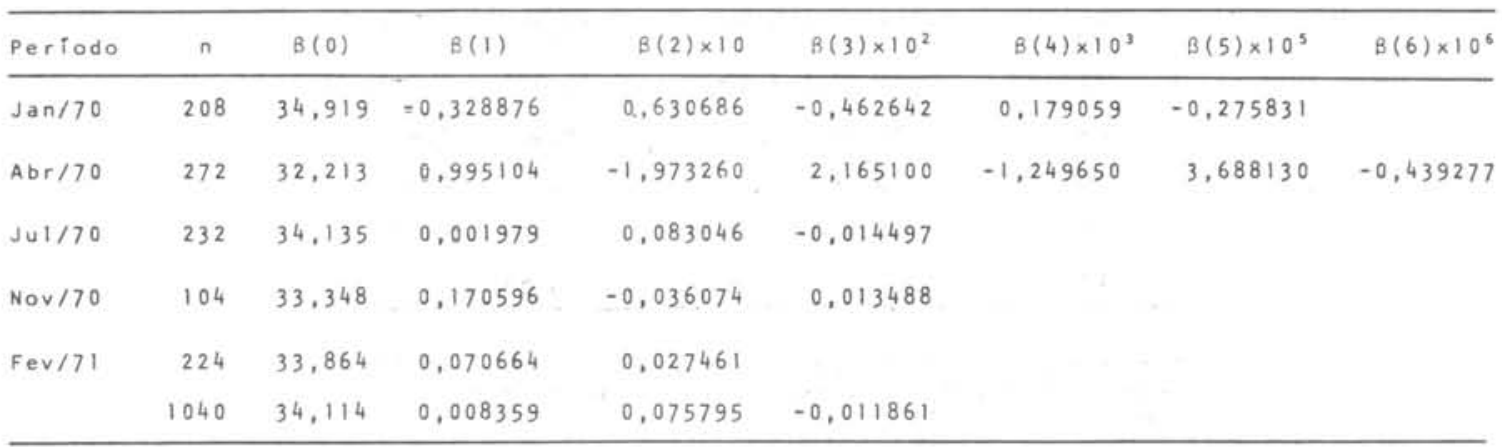




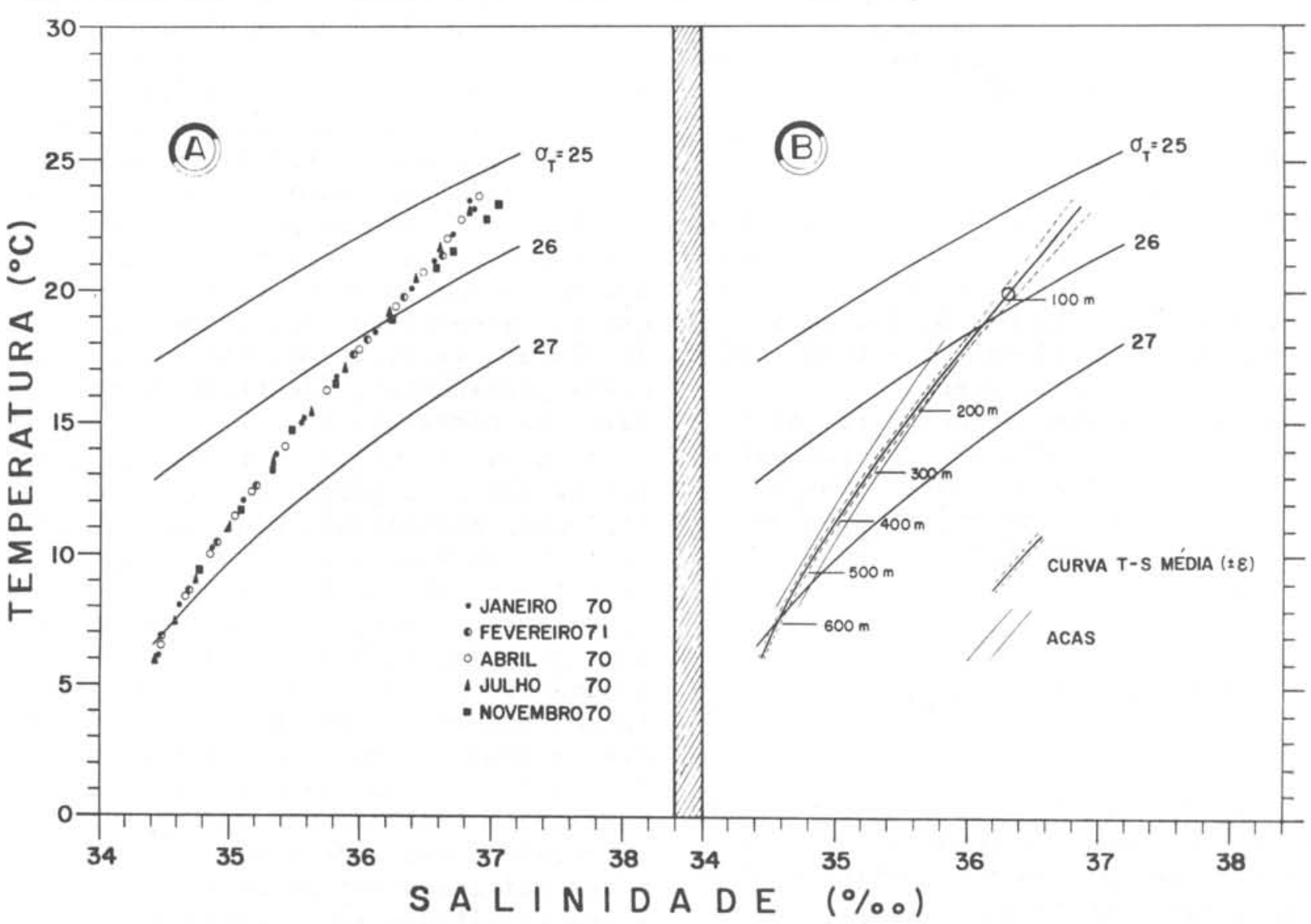

Fig. 5. Configuração de curvas T-S mëdias obtidas por ajuste polinomial. (A) alinhamento por um nümero discreto de pontos dos periodos indicados na legenda. (B) curva T-S mëdia do conjunto total de pontos selecionados e desvio médio quadrätico indicado pelas curvas tracejadas; a envoltöria extrema indica a ACAS definida por Sverdrup et al. (1942; p. 741). Os pontos foram selecionados entre a base da camada de mistura e a profundidade aproximada de $600 \mathrm{~m}$.

Tabela 3. Valores da salinidade mëdia da Massa de Ägua Central do Atlântico Sul (ACAS), para diferentes valores de temperatura

\begin{tabular}{ccc}
\hline Temperature & Salinidade ${ }^{*}( \pm d)$ & Salinidade $( \pm \varepsilon)$ \\
\hline${ }^{\circ} \mathrm{C}$ & $\%$ & $\%$ \\
8 & $34,64 \pm 0,08$ & $34,605 \pm 0,019$ \\
10 & $34,86 \pm 0,08$ & $34,837 \pm 0,018$ \\
12 & $35,11 \pm 0,08$ & $35,101 \pm 0,018$ \\
14 & $35,37 \pm 0,09$ & $35,391 \pm 0,024$ \\
16 & $35,64 \pm 0,10$ & $35,702 \pm 0,021$ \\
18 & $35,90 \pm 0,11$ & $36,028 \pm 0,025$ \\
20 & & $36,364 \pm 0,055$ \\
\hline
\end{tabular}

- De acordo com sverdrup et al. (1942, p. 741 e 743 ), médio. 
igual a $36,364 \pm 0,055$. Portanto, respeitando-se a proposição de trabalhos anteriores de carāter regional (Emílsson, 1961) e em grande escala (Mamayev, op. cit.), pode-se razoavelmente especular que, para a região em estudo um par $(\mathrm{S}, \mathrm{T})$ possa ser classificado de Massa de Agua Tropical (AT), sempre que $T \geq$ $20{ }^{\circ} \mathrm{C}$ e $\mathrm{S} \geq 36,36 \%$, e que esses vālores passam a representar os índices termohalinos da ACAS nos cálculos de percentagem de mistura de diferentes massas de água.

As variáveis dependentes da água do mar, numa coluna de água, são calculadas a partir de medições "in situ" das variáveis independentes $\mathrm{S}$ e T. Quando, por algum motivo, os valores de S apresentam falhas ou quando é amostrada apenas a estrutura térmica, torna-se necessārio utilizar um método alternativo para estimativas da salinidade. Como ficou demonstrado que a curva T-S tem uma configuração quase estacionária no período considerado (janeiro/70 a fevereiro/71), a expressão polinomial $\mathrm{S}=\mathrm{S}(\mathrm{T})$ das condições médias desse período, pode ser utilizada para estimativa da salinidade a partir do conhecimento da temperatura "in situ", $T=T(z)$, com um erro médio quadrático variável entre $\pm 0,02$ e $\pm 0,06 \%$. Esses resultados são concordantes aos obtidos por Stommel (1947), que indica incertezas entre $\pm 0,04$ e $\pm 0,05 \%$, nas estimativas da salinidade à partir de uma curva T-S média obtida para estações oceanográficas do Oceano Atlântico Norte.

As curvas T-S médias, representativas para as condições das águas da plataforma continental, foram calculadas com base nos diagramas T-S estatítico-volumétricos de cada um dos períodos de observação (Miranda, 1982). Os resultados obtidos, representados por um número discreto de pontos e considerando-se apenas temperaturas inferiores a $25^{\circ} \mathrm{C}$, são apresentados na Figura 6 .

A distribuição dos pontos para os diferentes períodos de observação apresenta, para densidades maiores do que 26,0 $\mathrm{g} / 1$, um espalhamento que pode ser considerado quase estacionário; esta condição corresponde a valores de $\mathrm{T}$ e $\mathrm{S}$ menores do que $18,0{ }^{\circ} \mathrm{C}$, e $36,0 \%$, respectivamente. Para densidade menor do que 26,0 $\mathrm{g} / 1$, a configuração das curvas T-S é diferente para os períodos de observação, indicando, por conseguinte, a sua natu- reza não estacionária,

A expressão polinomial de $S=S(T)$, ajustada com o conjunto de pontos da Figur:a 6 , é dada por:

$\mathrm{S}(\mathrm{T})=35,352-0,250883 \mathrm{~T}+0,0265952 \mathrm{~T}^{2}-$

$-0,59972 \times 10^{-3} \mathrm{~T}^{3}$

Essa equação é válida para o intervalo de variaçáo da temperatura de 10 a $25^{\circ} \mathrm{C}$ e representa analiticamente, em primeira aproximação, a curva T-S média das āguas da plataforma continental.

A curva que representa geometricamente a relação funcional dada pela equação (3) é apresentada na Figura 7. Nessa Figura, verifica-se que a parte quase estacionária da curva T-S $\left(\sigma_{\mathrm{T}}>26,0\right.$ $\mathrm{g} / 1)$, das águas da plataforma continental (H < $200 \mathrm{~m})$, é praticamente coincidente com a curva T-S média da ACAS presente na troposfera da região oceânica adjacente, à profundidade de até 300$400 \mathrm{~m}$. Portanto, temos a confirmação de que as águas da plataforma continental são oriundas dessas profundidades, em concordância com resultados de pesquisas anteriores realizadas em águas adjacentes à Ilha de Cabo Frio (Okuda, 1962; Silva, 1973; Mendonça, 1974; dentre outros) que mostram que as águas da plataforma continental têm comprovadamente origens profundas e que podem ser classificadas como ACAS.

\section{Derivada termohalina}

A derivada termohalina, dS/dT, em um ponto de uma curva T-S é interpretada geometricamente como igual ao inverso do coeficiente angular dessa curva. Essa interpretação decorre do fato de que, tradicionalmente, o diagrama T-S fornece a configuração geométrica da relação funcional $\mathrm{T}=\mathrm{T}(\mathrm{S})$; e não, $\mathrm{S}=\mathrm{S}(\mathrm{T})$.

A derivada termohalina pode ser colocada em função dos coeficientes de expansão térmica $(\alpha)$ e de contração salina (B). Com efeito, seja (S, T) um ponto genérico de uma curva T-S, no qual passa uma isolinha de volume específico à pressão atmosférica $\overline{\mathrm{V}}^{\circ}(\mathrm{S}, \mathrm{T})=$ cte..$]$; a equação dessa isolinha è dadā por

$$
\left(\frac{\partial V^{\circ}}{\partial S}\right)_{T} d S+\left(\frac{\partial V^{\circ}}{\partial T}\right)_{S} d T=0 .
$$




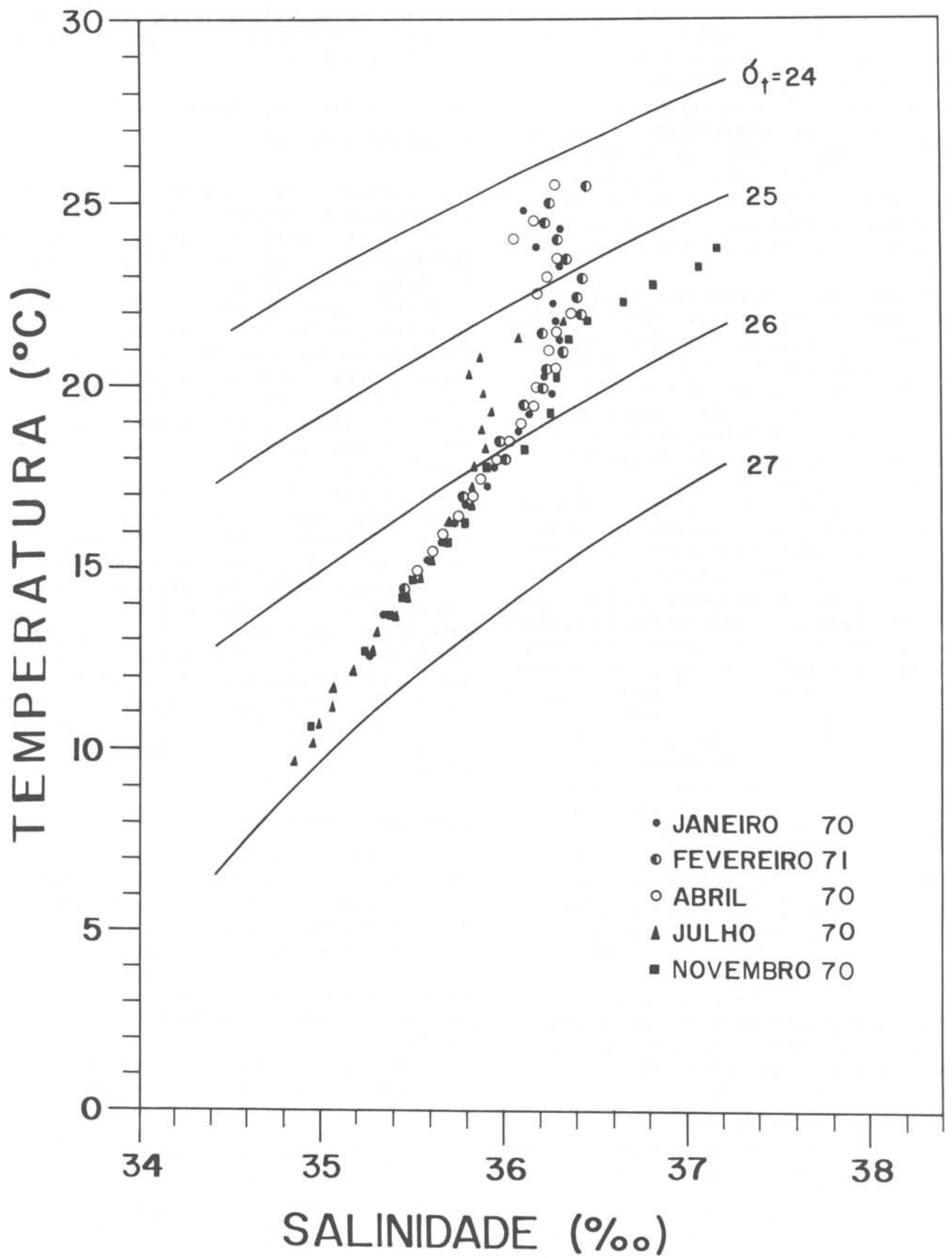

Fig. 6. Curva T-S mëdia das äguas da plataforma continental, obtida a partir dos diagramas T-S estatístico-volumétricos de cada um dos períodos de observação. 


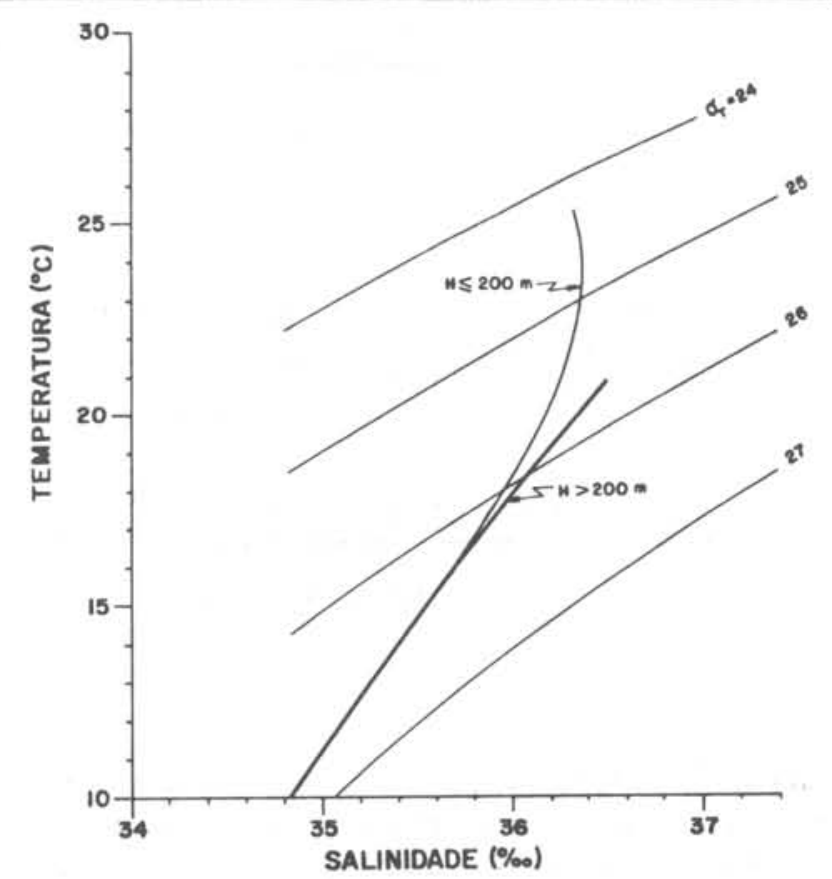

Fig. 7. Curvas T-S mëdias das äguas da plataforma continental $(\mathrm{H}<200 \mathrm{~m})$ e da camada superior da troposfera (ACAS) da região oceânica adjacente $(\mathrm{H}>200 \mathrm{~m})$.
Portanto, a derivada termohalina no ponto $(S, T)$ é,

$$
\frac{d S}{d T}=-\frac{\left(\frac{\partial V^{\circ}}{\partial T}\right)_{S}}{\left(\frac{\partial V^{\circ}}{\partial S}\right)_{T}} .
$$

Pelas definições dos coeficiente $\ell$ e $\beta$, pode-se escrever,

$$
\left(\frac{\partial V^{\circ}}{\partial T}\right)_{S}=\alpha V^{\circ} \quad \text { e } \quad\left(\frac{\partial V^{\circ}}{\partial S}\right)_{T}=-\beta V^{\circ} .
$$

Combinando-se as equações (5) e (6) segue-se,

$$
\frac{\mathrm{dS}}{\mathrm{dT}}=\frac{\alpha}{\beta}
$$

$\frac{\alpha}{\beta} \frac{\mathrm{dT}}{\mathrm{dS}}=1$.

A igualdade indicada na equação acima foi obtida para a derivada termohalina sobre uma isopicnal. Logo, se o primeiro membro dessa equação é calculado ao longo de uma curva T-S qualquer, o valor igual a 1 somente será obtido na condição em que essa massa de água é formada por processos de mistura isopicnal. Uma equação equivalente à (8) foi obtida por Pingree (1972), ao pesquisar o mecanismo segundo o qual os processos de mistura em pequena escala influenciam os de grande escala; nesse estudo, essa equação é obtida por integração das equações de conservação de calor e de sal, aplicadas às flutuações turbulentas de temperatura e de salinidade.

Para o caso de mistura não isopicnal, o primeiro membro da equação (8) foi denominado por Ingham (1966) de "razão de densidade constante" e denotado por $R_{\rho}$. Logo

$\frac{\beta}{\alpha} \frac{\mathrm{dS}}{\mathrm{dT}}=\left(\mathrm{R}_{\rho}\right)^{-1}$.

Inghan (op. cit.) determinou a quantidade $\mathrm{R}_{\rho}$ para a Massa de Água Central dos oceanos e elaborou mapas de sua distribuição até cerca de $1000 \mathrm{~m}$ de profundidade. No decorrer de sua pesquisa, ele notou que muitas curvas T-S dessa massa de água são melhor descritas por curva com $\mathrm{R}_{\mathrm{O}}=$ cte.

A condição $R_{\rho}=$ cte para Massas de Água Centrais dos oceanos Atlântico, Pacífico e Indico foi demonstrada quantitativamente por Schmitt (1981). Nessa pesquisa, a relação funcional $\mathrm{T}=\mathrm{T}(\mathrm{S})$, $\underset{\sim}{\operatorname{com}} \mathrm{R}_{\rho_{-}}=$cte, foi gerada por integração numérica da equação diferencial (9). 0 melhor resultado da concordância entre os valores experimentais de $\mathrm{T}$ e aqueles previstos teoricamente por essa integração foi obtido por sucessivos ajustes do valor $R_{\rho}$ e da constante de integração $T\left(S_{0}\right)$, onde $\mathrm{S}_{\mathrm{O}} \overline{\mathrm{e}}$ um valor constante de salinidade. Os valores de $\mathrm{R}_{\rho}$ calculados para as águas Centrais' desses oceanos variaram entre 1,59 e 3,82. Em particular, com dados de uma estação oceanográfica do Atlântico Sul (Lat. 
$17^{\circ} 02^{\prime} \mathrm{S}$; Long. $\left.031^{\circ} 00^{\prime} \mathrm{W}\right)$ foi determinado o valor $\mathrm{R}_{\rho}=1,894$, ○ qual é considerado representativo para as características termohalinas da ACAS dessa região.

Dos resultados precedentes, seguemse as seguintes expressões para o cālculo da derivada termohalina:

$$
\frac{\mathrm{dS}}{\mathrm{dT}}=\frac{\alpha}{\beta \mathrm{R}_{\rho}} \text {, }
$$

e

$$
\begin{aligned}
\frac{\mathrm{dS}}{\mathrm{dT}}= & 8,359 \times 10^{-3}+0,015159 \mathrm{~T}- \\
& -0,035583 \times 10^{-2} \mathrm{~T}^{2} .
\end{aligned}
$$

A equação (10) foi obtida a partir da equação (9), sendo uma função dos coeficientes de dilatação volumétrica e de contração salina e, portanto, das variáveis (S, T, p). A equação (11) é a derivada da expressão polinomial média de $\mathrm{S}=\mathrm{S}(\mathrm{T})$, cujos coeficientes são apresentados na Tabela 2. Logo, a comparação dos valores da derivada termohalina obtida por essas equações constitui uma maneira para a determinação de $R_{\rho}$, com ajustes sucessivos desse coeficiente, de forma a minimizar os desvios dessa comparação.

Os valores de dS/dT, para as condições médias obtidas da variáveis $S$ e $T$, foram calculados pela equação (10). Os coeficientes $\alpha$ e $\beta$ foram determinados pela equação de estado de Chen \& Millero (1977), equação esta tambēm utilizada por Schmitt (1981) para a integração numérica da equação (9). Utilizando-se a notação do trabalho original e a forma geral da equação de estado de Chen \& Millero (op. cit.), os coeficientes de expansão térmica e de contração salina, derivados dessa equação, são expressos por:

$\alpha=\frac{1}{V^{p}}\left[\left(\partial V^{\circ} / \partial T\right)_{S, p}-\frac{p\left(\partial V^{\circ} / \partial T\right) S, p}{K^{\circ}+A p+B p^{2}}\right.$

$$
\left.+\mathrm{pV}^{\circ} \frac{\left(\partial \mathrm{K}^{\circ} / \partial \mathrm{T}\right)_{\mathrm{S}, \mathrm{p}}+\mathrm{p}(\partial \mathrm{A} / \partial \mathrm{T}) \mathrm{S}, \mathrm{p}}{\left(\mathrm{K}^{\circ}+\mathrm{Ap}+\mathrm{Bp}^{2}\right)^{2}}\right]
$$

$$
\begin{aligned}
& B=-\frac{1}{\mathrm{~V}^{\mathrm{p}}}\left[\left(\partial \mathrm{V}^{\circ} / \partial \mathrm{S}\right)_{\mathrm{T}, \mathrm{p}}-\frac{\mathrm{p}\left(\partial \mathrm{V}^{\circ} / \partial \mathrm{S}\right)_{\mathrm{T}, \mathrm{p}}}{\mathrm{K}^{\circ}+\mathrm{Ap}+\mathrm{Bp}^{2}}\right. \\
& \left.+p V^{\circ} \frac{\left(\partial K^{\circ} / \partial S\right) T, p+p(\partial A / \partial S)_{T, p}}{\left(K^{\circ}+A p+B p^{2}\right)^{2}}\right] \text {. }
\end{aligned}
$$

onde $V^{\circ}$ e $V^{p}$ são os volumes específicos sob pressão atmosférica e à pressão de $\underline{\mathrm{p}}$ bärias, respectivamente. $\mathrm{V}^{\circ}, \mathrm{K}^{\circ}$ e A são parâmetros funções da temperatura (T) e da salinidade (S), cujas expressões algébricas são dadas no trabalho mencionado. As equações (12) e (13) são vālidas para as condições oceânicas $\left(30\right.$ a $40 \%$ o,-2 a $38^{\circ} \mathrm{C}$ e até 1.000 bárias de pressão).

Os resultados obtidos pelas equações (10) e (11), são apresentados

na Tabela 4. A melhor concordância entre os valores da derivada termohalina, que minimizou a diferença entre os resultados dessas equações, foi obtido com $R_{\rho}=1,873$, resultado este muito próximo ao obtido por Schmitt (1981), ou seja, $R_{\rho}=1,894$.

Tabela 4. Derivada termohalina calculada com as equações (10) e (11), utilizando condições mëdias da curva T-S

\begin{tabular}{ccccc}
\hline $\begin{array}{c}P \\
(d b)\end{array}$ & $\left.{ }^{\circ} \mathrm{C}\right)$ & $(\% / 00)$ & $R_{P}=1,873$ & $\begin{array}{c}d S / d T \\
S=S(T)\end{array}$ \\
\hline 100 & 19,49 & 36,278 & 0,183 & 0,169 \\
151 & 17,10 & 35,880 & 0,168 & 0,164 \\
201 & 15,35 & 35,599 & 0,156 & 0,157 \\
251 & 14,10 & 35,406 & 0,148 & 0,151 \\
302 & 13,08 & 35,255 & 0,142 & 0,146 \\
352 & 12,15 & 35,122 & 0,136 & 0,140 \\
402 & 11,24 & 34,997 & 0,130 & 0,134 \\
453 & 10,25 & 34,868 & 0,123 & 0,126 \\
503 & 9,30 & 34,753 & 0,117 & 0,118 \\
553 & 8,25 & 34,630 & 0,100 & 0,109 \\
604 & 7,35 & 34,538 & 0,104 & 0,101 \\
\hline
\end{tabular}


Na Tabela 4, verifica-se que os valores da derivada termohalina são muito próximos entre si, com a maior diferença sendo observada à pressão de $100 \mathrm{db}$.

Schmitt (1979) demonstrou que o parâmetro $R_{\rho} \quad \bar{e}$ o que determina a intensidade da difusão de sal pelo processo de mistura conhecido como "salt fingers". Com base na constância desse parâmetro, Schmitt (1981) propõe um mecanismo simples de dupla difusão, para explicar a maior atividade do processo mencionado. Nesse trabalho, com base na teoria e na experiência, ele conclui que a confifuração das curvas T-S em grande esca-

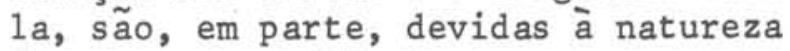
dos processos difusivos em pequena escala. Logo, a boa concordância da curva T-S das Massas de Āgua Centrais com a curva teórica prevista com $\mathrm{R}_{0}=$ cte, fornece evidências de que a mistura através dos "salt fingers" desempenha um papel significativo na formação dessas massas de água.

\section{Conclusões}

Com base nas características da correlação T-S das águas das camadas de mistura e subjacente, e considerando-se também resultados e sugestões de trabalhos anteriores, fica comprovado que as Massas de Ãgua Subtropical Profunda (ASTP), Central do Atlântico Sul (ACAS) e Subtropical (AST) constituem, para a região em estudo, diferentes designações de uma mesma massa de água. Com os resultados obtidos verifica-se que em correspondência ao valor de temperatura $20^{\circ} \mathrm{C}$, tem-se o valor de salinidade igual a $36,36 \%$ que é ligeiramente superior à salinidade do índice termohalino modificado da ACAS

$(36,20 \%$ \% ). Portanto, é razoāvel admitir-se o uso exclusivamente para a região em estudo desse novo índice termohalino quando dos cálculos de porcentagens de mistura de diferentes massas de āgua e para a identificação da Massa de Âgua Tropical.

As curvas T-S médias da ACAS presente na região são representadas analiticamente por polinômios que variam do $3^{\circ}$ ao $6^{\circ}$ grau. A representação gráfica desses polinômios são arcos de curva que apresentam um pequeno desvio sazonal, demonstrando que a configuração da ACAS apresenta-se bastante estáve1 no decorrer do ano. As curvas T-S médias da plataforma continental, determinados com os resultados da análise T-S estatístico-volumétrica, apresentam uma grande variação sazonal para as águas menos densas do que 26,0 (em unidades de Sigma-T); para as äguas mais densas, caracterizadas por valores de $\mathrm{S}$ e $\mathrm{T}$ inferiores a $36,0^{\circ} \%$ e $18,0^{\circ} \mathrm{C}$, a correlação T-S apresenta-se quase estacionária. Como esses valores definem o indice termohalino original da ACAS, temos uma evidência experimental da influência do processo advectivo (horizontal e vertical - ressurgência) para a renovação das águas da plataforma continental. A comparação das curvas T-S médias obtidas para as águas da plataforma continental e oceânicas, demonstra, em concordância com trabalhos anteriores, que as äguas da plataforma continental têm predominantemente origem profunda (300 $400 \mathrm{~m}$ ).

Os valores da derivada termohalina, $\mathrm{dS} / \mathrm{dT}$, obtidos diretamente pela derivação da expressão polinomial da curva T-S média da ACAS, são praticamente iguais aos correspondentes valores da expressão teórica que define essa quantidade em função dos coeficientes de expansão térmica e de contração salina, e da razão de densidade constante. Conseqüentemente, essa forma da curva T-S média para a área em estudo pode ser utilizada para estimativas da salinidade com um erro médio quadrático não superior a $\pm 0,06 \%$.

Com o método alternativo para a determinação da razão de densidade constante, $R_{\rho}$, foi obtido o valor 1,873 , mostrando conclusivamente que as características termohalinas dessa massa de água são geradas por processos de mistura não isopicnal. Esse valor é 0,02 inferior ao obtido para as condições da ACAS da costa leste do Brasil, com um método interativo de integração numérica.

\section{Resumo}

As variações na estrutura e em propriedades de massas de água da plataforma continental e das de àgua oceânica adjacente são estudadas com base em expressões analíticas das curvas T-S. As medições das variáveis oceanogrāficas foram feitas por métodos classicos numa rede de estações hidrogräficas, as quais foram amostradas em cin- 
co intervalos de tempo (5-10 dias) cada um, entre os meses de janeiro de 1970 e fevereiro de 1971.

A revisão da classificação das massas de água usada sob condições regionais confirma que as Massas de Âgua Subtropical Profunda (ASTP), Subtropical (AST) e Central do Atlântico Sul (ACAS), são designações diferentes de uma mesma massa de água. A última nomenclatura, Āgua Central do Atlântico Sul (ACAS), è sugerida para indicar a massa de água com indice termohalino $\left(20,0^{\circ} \mathrm{C} ; 36,36^{\circ} \%\right.$ o $)$, a qual é observada sob a Massa de Āgua Tropical. Esse valor de salinidade é um pouco maior do que o índice termohalino modificado da ACAS $(36,20 \%$ ) e pode ser usado com vantagem nos cálculos volumétricos na ärea.

As expressões polinomiais da curva $\mathrm{T}-\mathrm{S}$, cujos coeficientes foram determinados pelo método dos mínimos quadrados, foram usadas em aplicações práticas. Nessas aplicações, foram desenvolvidos métodos alternativos para.a determinação da derivada termohalina e da razão de densidade constante $\left(\mathrm{R}_{\rho}=\alpha \mathrm{dT} / \beta \mathrm{dS}\right)$.

\section{Referências bibliográficas}

ALME IDA, E. G. de \& AZEVEDO, M. A. L. de 1970. Comparação entre os dados co1hidos pelos sensores remotos na ârea de teste Cabo Frio com um modelo matemático da ressurgência. Anais hidrogr., Rio de J., 37 (DH 3-27): 249-260.

AZEVEDO, M. A. L. de 1974. Determinação de um único valor do volume específico médio para toda a costa do Brasil até Mar del Plata. Anais hidrogr., Rio de J., 31 (DH 3-31): 135-144.

BERNARDI Jr, H. 1969. Estudo preliminar de um método estatístico para controle de dados oceanográficos. Anais hidrogr., Rio de J., 26(DH 3-26):165-174.

CHEN, C. T. \& MILLERO, F. J. 1977. Precise equation of state of seawater for oceanic ranges of salinity, temperature and pressure. Deep-Sea Res., 24:365369.
DEACON, G. E. R. 1933. A general account of the hydrology of the South Atlantic Ocean. Discovery Rep., 7:171-238.

1937. The

hydrology of the Southern Ocean. Discovery Rep., 15:1-124.

EMÍlsSON, I. 1959. Alguns aspectos físicos e químicos das äguas marinhas brasileiras. Ciênc. Cult., S Paulo, 11(2):44-54.

\section{The shelf and}

coastal waters off southern

Brazil. Bolm Inst. oceanogr.,

S Paulo, 11(2):101-112.

IKEDA, Y.; MIRANDA, L. B. de \& ROCK, N. J. 1974. Observations on stages of upwelling in the region of Cabo Frio (Brazil) as conducted by continuous surface temperature and salinity measurements. Bolm Inst. oceanogr., S Paulo, 23:33-46.

INGHAM, M. C. 1966. The salinity extrema of the World Ocean. Ph.D. Dissertation. Oregon State University, Corvallis, 63p.

MAGLIOCCA, A.; MIRANDA; L. B. de \& SIGNORINI, S. R. 1979. Physical and chemical aspects of transient stages of the upwelling at southwest of Cabo Frio (Lat. $23^{\circ} \mathrm{S}$ Long. $\left.42^{\circ} \mathrm{W}\right)$. Bolm Inst. oceanogr., S Paulo, 28(2):37-46.

MAMAYEV, O. I. 1975. Temperaturesalinity analysis of world ocean waters. Amsterdam, E1sevier, 374p.

MASCARENHAS Jr, A. S. da; MIRANDA, L. B. de \& ROCK, N. J. 1971. A study of the oceanographic conditions in the region of Cabo Frio. In: Costlow Jr, J. D., ed. - Fertility of the sea. New York, Gordon \& Breach, v. 1, p. 285-308.

MENDONÇA, C. da F. 1974. Características mais freqüentes das massas de água da região de Cabo Frio e comprovação da origem profunda da água litoral. Publções Inst. Pesq. Mar., (080):1-29. 
MESQUITA, A. R. de; LEITE, J. B. \& RIZZO, R. 1979. Contribuição ao estudo das correntes marinhas na plataforma entre Cabo Frio e Cananéia. Bolm Inst. oceanogr., S Paulo, 28(2):95-100.

MIRANDA, L. B. de 1982. Anälise de massas de água da plataforma continental e da região oceânica adjacente: Cabo de São Tomé (RJ) e Ilha de São Sebastião (SP). Tese de Livre-Docência. Universidade de São Paulo, Instituto Oceanogräfico, 123p. + figs.

OKUDA, T. 1962. Physical and chemical oceanography over continental shelf between Cabo Frio and Vitoria (Central Brazil). J. oceanogr. Soc. Japan, 20th annivers. vol.:514-540.

PINGREE, R. D. 1972. Mixing in the deep stratified ocean. Deep-Sea Res., 19:549-561.

RODRIGUES, R. F. 1977. Evolução da nhassa d'água durante a ressurgência em Cabo Frio. Publções Inst. Pesq. Mar., (115): 1-31.

SCHMITT, R. W. 1981. Form of the temperature-salinity relationship in the Central Water: evidence for double-diffusive mixing. J. phys . Oceanogr., (11):1015-1026.

SCHMITT, Jr, R. W. 1979. The growth rate of super-critical salt fingers. Deep-Sea Res., 26:23-40.
SILVA, P. de C. M. da 1959. Oceanografia do triângulo Cabo Frio, Trindade e Salvador. Anais hidrogr., Rio de J., 16:213-308.

1973. A ressurgência em Cabo Frio (I). Publções Inst. Pesq. Mar., (78):1-55.

\& RODRIGUES, R.

F. 1966. Modificações da estrutura vertical das águas sobre a borda da plataforma continental, por influência do vento. Notas téc. Inst. Pesq. Mar., (35):1-13.

STOMMEL, H. 1947. Note on use of the T-S correlation for dynamic height anomaly computations. J. mar. Res., 6(2): 85-92.

SVERDRUP, H. U.; JOHNSON, M. W. \&

FLEMING, R. H. 1942. The oceans; their physics, chemistry and, general biology. Englewood Cliffs, N. J., Prentice Hall, 1087p.

TANAKA, K. 1977. Simulação da ressurgência comparada com dados oceanográficos e de sensores remotos em Cabo Frio. Anais hidrogr., Rio de J., 34 (DH 3-34):169-321.

THOMSEN, H. 1962. Masas de água caracteristicas del Oceano Atlantico-Parte Sudoeste. Serv. Hidrogr. Nava1, B Aires, Público, (H-632): 1-27.

(Recebido em 01-07-1983; aceito em 29-11-1985) 